PROCEEDINGS OF THE

AMERICAN MATHEMATICAL SOCIETY

Volume 126, Number 9, September 1998, Pages 2733-2736

S $0002-9939(98) 04460-8$

\title{
THE EQUIVALENCE OF SOME BERNOULLI CONVOLUTIONS TO LEBESGUE MEASURE
}

\author{
R. DANIEL MAULDIN AND KÁROLY SIMON \\ (Communicated by Frederick W. Gehring)
}

\begin{abstract}
Since the 1930's many authors have studied the distribution $\nu_{\lambda}$ of the random series $Y_{\lambda}=\sum \pm \lambda^{n}$ where the signs are chosen independently with probability $(1 / 2,1 / 2)$ and $0<\lambda<1$. Solomyak recently proved that for almost every $\lambda \in\left[\frac{1}{2}, 1\right]$, the distribution $\nu_{\lambda}$ is absolutely continuous with respect to Lebesgue measure. In this paper we prove that $\nu_{\lambda}$ is even equivalent to Lebesgue measure for almost all $\lambda \in\left[\frac{1}{2}, 1\right]$.
\end{abstract}

\section{INTRODUCTION}

For each $\lambda \in(0,1)$ we define the random variable

$$
Y_{\lambda}=\sum_{n=0}^{\infty} \theta_{n} \cdot \lambda^{n}
$$

where $\theta_{n}$ are independent random variables with $\operatorname{Prob}\left(\theta_{n}=-1\right)=\operatorname{Prob}\left(\theta_{n}=1\right)=$ $\frac{1}{2}$. The distribution $\nu_{\lambda}$ of $Y_{\lambda}$ is sometimes called a symmetric infinite Bernoulli convolution. One can easily see that for $0<\lambda<\frac{1}{2}$ the distribution $\nu_{\lambda}$ is supported on a Cantor set of zero Lebesgue measure. Since the 1930's a lot of work has been done to characterize $\nu_{\lambda}$ for $\frac{1}{2}<\lambda$ (for a good survey see e.g. Peres, Solomyak (1996a) [4]). Among these results the most interesting ones are as follows: P. Erdős (1939) [1] proved that $\nu_{\lambda}$ is singular with respect to Lebesgue measure, if $\lambda$ is the reciprocal of a PV number. (An algebraic integer is a PV number provided all of its conjugates are less than one in modulus.) On the other hand, Wintner (1935) [7] proved that $\nu_{\lambda}$ is absolutely continuous for $\lambda=2^{-\frac{1}{k}}$, for each $k \geq 1$, and Garsia (1962) [3] found some other algebric integers for which $\nu_{\lambda}$ is absolutely continuous. Moreover, Erdős (1940) [2] also proved that there exists $a<1$ such that the distribution $\nu_{\lambda}$ is absolutely continuous with respect to Lebesgue measure for (Lebesgue) a.e. $\lambda \in(a, 1)$. Then P. Erdős asked:

$$
\text { Is this statement true with } a=\frac{1}{2} ?
$$

Received by the editors February 11, 1997.

1991 Mathematics Subject Classification. Primary 26A30, 28A78, 28A80.

Key words and phrases. Bernoulli convolution, equivalent measures.

The first author's research was supported by NSF Grant DMS-9502952. The second author's research was partially supported by grants F19099 and T19104 from the OTKA Foundation.

(C)1998 American Mathematical Society 
This exciting problem remained open for more than fifty years. Then Solomyak (1995) [6] gave a positive answer (see also Peres, Solomyak (1996a) [4] for a shorter proof). Namely,

Theorem 1 (Solomyak).

$$
\nu_{\lambda} \ll m \text { for Lebesgue a.e. } \lambda \in\left(\frac{1}{2}, 1\right),
$$

where $m$ is Lebesgue measure.

Answering a problem of the first author, posed to the Conference on Fractals and Stochastics (1994, Finsterbergen), we prove that that $\nu_{\lambda}$ is even equivalent to Lebesgue measure for a.e. $\lambda \in\left[\frac{1}{2}, 1\right]$. Using Solomyak's theorem, it is enough to prove that Lebesgue measure is either absolutely continuous or singular with respect to $\nu_{\lambda}$ for each $\lambda$. Actually we prove this statement for a more general family of measures. Furthermore, Peres, Solomyak (1996b) [5] proved that if the probabilities of choosing the signs + and - in $Y_{\lambda}$ are $(p, 1-p)$ where $p \in[1 / 3,2 / 3]$, then $\nu_{\lambda} \ll m$ holds for a.e. $\lambda \in\left[p^{p}(1-p)^{1-p}, 1\right]$. Using this, it follows from our result that even in this non-symmetric case the distributions are not only absolutely continuous but equivalent to Lebesgue measure for a.e. $\lambda \in\left[p^{p}(1-p)^{1-p}, 1\right]$. (For smaller $\lambda$ the distributions are singular.)

We thank Yuval Peres for some useful conservations.

\section{Notation}

For an arbitrary $\lambda \in\left(\frac{1}{2}, 1\right)$ we define the 'projection' $\Pi_{\lambda}:\{-1,1\}^{\mathbf{N}} \rightarrow\left[\frac{-1}{1-\lambda}, \frac{1}{1-\lambda}\right]$ by $\Pi_{\lambda}(\mathbf{i})=\sum_{k=0}^{\infty} i_{k} \lambda^{k}$. Let $\mu$ be any Borel probability measure on $\{-1,1\}^{\mathbf{N}}$ for which

$$
\mu(B)>0 \Longrightarrow \mu\{(i, B)\}>0
$$

holds for all $B \subset\{-1,1\}^{\mathbf{N}}$ and $i \in\{-1,1\}$, where $(i, B):=\left\{(i, \mathbf{j}) \in\{-1,1\}^{\mathbf{N}}: \mathbf{j} \in\right.$ $B\}$. For example $\mu$ may be any Bernoulli measure on $\{-1,1\}^{\mathbf{N}}$ with probabilities $(p, 1-p), 0<p<1$. The 'push down measure' of $\mu$ is $\alpha_{\lambda, \mu}(B):=\mu\left(\Pi_{\lambda}^{-1}(B)\right)$. We denote the interval $\left[\frac{-1}{1-\lambda}, \frac{1}{1-\lambda}\right]$ by $I$. Further, we define $S_{i}: I \rightarrow I, S_{i}(x):=\lambda x+i$ for $(i=-1,1)$. The iterates of $S_{i}$ are

$$
S_{i_{1} \ldots i_{n}}(x):=S_{i_{1}} \circ \ldots \circ S_{i_{n}}(x) .
$$

The image of $I$ under $S_{i_{1} \ldots i_{n}}$ is called $I_{i_{1} \ldots i_{n}}$. The inverse of $S_{i_{1} \ldots i_{n}}$ is defined only on $I_{i_{1} \ldots i_{n}}$. So $S_{i_{1} \ldots i_{n}}^{-1}(A):=S_{i_{1} \ldots i_{n}}^{-1}\left(A \cap I_{i_{1} \ldots i_{n}}\right)$. Then $S_{i}^{-1}(x)=\frac{1}{\lambda} x-\frac{i}{\lambda}$ for $x \in I_{i}$ $(i=-1,1)$. We denote the Lebesgue measure of a set $A$ by $m(A)$.

\section{The Theorem And its CONSEQUences}

Theorem 2. Either $m \ll \alpha_{\lambda, \mu}$ or $m \perp \alpha_{\lambda, \mu}$.

If $\mu$ is the Bernoulli measure with probabilities $\left(\frac{1}{2}, \frac{1}{2}\right)$ then $\nu_{\lambda}=\alpha_{\lambda, \mu}$. Using Solomyak's Theorem, we obtain

Consequence 1. For almost all $\lambda \in\left(\frac{1}{2}, 1\right), \nu_{\lambda}$ is equivalent to Lebesgue measure.

Clearly, any Bernoulli measure $\mu$ with probabilities $(p, 1-p)$, satisfies (1) (if $p \neq 0)$. Thus, 
Consequence 2. Let $\eta_{\lambda}$ be the distribution of the random series $Z_{\lambda}=\sum \pm \lambda^{n}$, where the signs are are chosen independently with probabilities $(p, 1-p)$ and $0<$ $\lambda<1$. Then either $m \ll \eta_{\lambda}$ or $m \perp \eta_{\lambda}$.

Let $\eta_{\lambda}$ be as above. Then $\eta_{\lambda}$ is singular for all $\lambda<p^{p}(1-p)^{1-p}$ (see Peres, Solomyak (1996b) [5, Theorem 2 (a)]). Also Peres, Solomyak (1996b) [5, Corollary 1.4] proved that for $p \in[1 / 3,2 / 3]$ and for a.e. $\lambda \in\left[p^{p}(1-p)^{1-p}, 1\right], \eta_{\lambda} \ll m$. Thus, using our previous consequence we obtain

Consequence 3. Let $\eta_{\lambda}$ be the distribution of the random series $Z_{\lambda}=\sum \pm \lambda^{n}$, where the signs are are chosen independently with probabilities $(p, 1-p)$. Then for each $p \in[1 / 3,2 / 3]$ and for almost every $\lambda \in\left[p^{p}(1-p)^{1-p}, 1\right]$, the distribution $\eta_{\lambda}$ is equivalent to Lebesgue measure.

\section{LEMMAS AND PROOFS}

To prove Theorem 2 we need two lemmas.

Lemma 1. Let $A \subset I$. Then $\alpha_{\lambda, \mu}(A)=0 \Longrightarrow \alpha_{\lambda, \mu}\left(S_{i}^{-1}(A)\right)=0(i=-1,1)$.

Proof. First observe that

$$
\Pi_{\lambda}^{-1}(A)=\left\{\left(-1, \Pi_{\lambda}^{-1}\left(S_{-1}^{-1}(A)\right)\right)\right\} \bigcup\left\{\left(1, \Pi_{\lambda}^{-1}\left(S_{1}^{-1}(A)\right)\right)\right\} .
$$

This is so, since for $i=-1,1$

$$
\begin{aligned}
\mathbf{j} \in \Pi_{\lambda}^{-1}\left(S_{i}^{-1}(A)\right) & \Longleftrightarrow \sum_{k=0}^{\infty} j_{k} \lambda^{k} \in S_{i}^{-1}(A) \Longleftrightarrow \sum_{k=0}^{\infty} j_{k} \lambda^{k} \in \frac{1}{\lambda} A-\frac{i}{\lambda} \\
& \Longleftrightarrow i+\sum_{k=0}^{\infty} j_{k} \lambda^{k+1} \in A \Longleftrightarrow(i, \mathbf{j}) \in \Pi_{\lambda}^{-1}(A) .
\end{aligned}
$$

To get a contradiction we assume that there exists a set $A$ such that $\alpha_{\lambda, \mu}(A)=0$ and $\alpha_{\lambda, \mu}\left(S_{i}^{-1}(A)\right)=\mu\left(\Pi_{\lambda}^{-1}\left(S_{i}^{-1}(A)\right)\right)>0$ holds for some $i \in\{-1,1\}$.

Then from (1), it follows that $\mu\left(\left(i, \Pi_{\lambda}^{-1}\left(S_{i}^{-1}(A)\right)\right)\right)>0$. Using (2), we find that $\mu\left(\Pi_{\lambda}^{-1}(A)\right)=\alpha_{\lambda, \mu}(A)>0$. This contradiction proves our lemma.

Let $C \subset I$ be an arbitrary fixed Borel set. Let $C_{0}:=C$ and

$$
C_{-(k+1)}:=\left(S_{-1}^{-1}\left(C_{-k}\right) \cup S_{1}^{-1}\left(C_{-k}\right)\right) .
$$

Then the 'backward orbit' of $C$ in $I$ is

$$
\Lambda_{-}:=\bigcup_{k \geq 0} C_{-k} .
$$

Lemma 2. For any $C \subset I$, the set $\Lambda_{-}$defined above is either a set of zero measure or a full measure subset of I with respect to Lebesgue measure.

Proof. Let $\bar{\Lambda}_{-}:=I \backslash \Lambda_{-}$. Obviously, it is enough to prove the statement of Lemma 2 for the set $\bar{\Lambda}_{-}$instead of $\Lambda_{-}$. Observe that

$$
x \in \bar{\Lambda}_{-} \Longrightarrow S_{i}(x) \in \bar{\Lambda}_{-}
$$

holds, since $S_{i}(x) \notin \bar{\Lambda}_{-} \Longrightarrow \exists k \geq 0$ such that $S_{i}(x) \in C_{-k} \cap I_{i}$. Then $x=$ $S_{i}^{-1}\left(S_{i}(x)\right) \in C_{-(k+1)} \subset \Lambda_{-}$. Iterate (4) to obtain

$$
S_{i_{1} \ldots i_{n}}\left(\bar{\Lambda}_{-}\right) \subset \bar{\Lambda}_{-},
$$


for each $n \in \mathbf{N}$ and $\left(i_{1}, \ldots, i_{n}\right) \in\{-1,1\}^{n}$. Suppose that $m\left(\bar{\Lambda}_{-}\right)>0$. Then $d:=$ $\frac{m\left(\bar{\Lambda}_{-}\right)}{|I|}$ is positive. Using $(5)$, we obtain that $m\left(\bar{\Lambda}_{-} \cap I_{i_{1} \ldots i_{n}}\right) \geq m\left(S_{i_{1} \ldots i_{n}}\left(\bar{\Lambda}_{-}\right)\right)=$ $\lambda^{n} \cdot d \cdot|I|$. Thus

$$
\frac{m\left(\bar{\Lambda}_{-} \cap I_{i_{1} \ldots i_{n}}\right)}{\left|I_{i_{1} \ldots i_{n}}\right|} \geq d
$$

holds for each $i_{1} \ldots i_{n}$.

On the other hand, let $J \subset I$ be an arbitrary interval. Then we can find $n$ and $i_{1} \ldots i_{n}$ such that $I_{i_{1} \ldots i_{n}} \subset J$ and

$$
\frac{\left|I_{i_{1} \ldots i_{n}}\right|}{|J|} \geq \frac{\lambda}{3}
$$

Now, from (6) and (7) together, it follows that

$$
\frac{m\left(\bar{\Lambda}_{-} \cap J\right)}{|J|} \geq d \cdot \frac{\lambda}{3} .
$$

That is, $\Lambda_{-}$has no density point. Thus $\bar{\Lambda}_{-}$is a full measure subset of $I$. This completes the proof of Lemma 2.

Proof of Theorem 2. Suppose that $m \nless \alpha_{\lambda, \mu}$. Then there is a set $C \subset I$ such that $m(C)>0$ and $\alpha_{\lambda, \mu}(C)=0$. Define $\Lambda_{-}$by (3). Then $m\left(\Lambda_{-}\right)>0$; thus it follows from Lemma 2 that $\Lambda_{-}$is a full measure subset of $I$ with respect to Lebesgue measure. On the other hand, Lemma 1 implies that $\alpha_{\lambda, \mu}\left(\Lambda_{-}\right)=0$. So $m \perp \alpha_{\lambda, \mu}$. This completes the proof of Theorem 2 .

\section{REFERENCES}

[1] P.Erdős (1939). On a family of symmetric Bernoulli convolutions, Amer. J. Math. 61, 974-976. MR 1:52a

[2] P.Erdős (1940). On the smoothness properties of a family of Bernoulli convolutions, Amer. J. Math. 62, 180-186. MR 1:139e

[3] A.M. Garsia (1962). Arithmetic properties of Bernoulli convolutions, Trans. Amer. Math. Soc. 102, 409-432. MR 25:1409

[4] Y. Peres and B. Solomyak (1996a). Absolute continuity of Bernoulli convolutions, Math. Research Letters 3:2, 231-239. MR 97f:28006

[5] Y. Peres and B. Solomyak (1996b). Self-similar measures and intersections of Cantor sets, Trans. Amer. Math. Soc., to appear.

[6] B. Solomyak (1995). On the random series $\sum \pm \lambda^{i}$ (an Erdős problem), Annals of Math. 142, 611-625. MR 97d:11125

[7] A. Wintner (1935). On convergent Poisson convolutions, Amer. J. Math. 57, 827-838.

Department of Mathematics, P. O. Box 305118, University of North Texas, Denton, TEXAS 76203-5118

E-mail address: mauldin@dynamics.math.unt.edu

Current address, K. Simon: Institute of Mathematics, University of Miskolc, Miskolc-Egyetemvaros, H-3515 Hungary

E-mail address: matsimon@gold.uni-miskolc.hu 